\title{
Epidemiology of malaria in a village in the Rufiji River Delta, Tanzania: declining transmission over 25 years revealed by different parasitological metrics
}

Anna Färnert ${ }^{1 *}$, Victor Yman ${ }^{1}$, Manijeh Vafa Homann ${ }^{1}$, Grace Wandell ${ }^{1}$, Leah Mhoja ${ }^{2}$, Marita Johansson², Salome Jesaja ${ }^{2}$, Johanna Sandlund ${ }^{1}$, Kazuyuki Tanabe ${ }^{3 \wedge}$, Ulf Hammar ${ }^{4}$, Matteo Bottai ${ }^{4}$, Zulfiqarali G Premji ${ }^{5,6}$, Anders Björkman ${ }^{7}$ and Ingegerd Rooth ${ }^{1,2}$

\begin{abstract}
Background: Assessments of the epidemiology of malaria over time are needed to understand changes in transmission and guide control and elimination strategies.

Methods: A longitudinal population study was established in 1985 in Nyamisati village in the Rufiji River Delta, Tanzania. A physician and research team lived in the village 1984-2000. Parasite prevalence by microscopy and two PCR methods, spleen rates and haemoglobin levels were measured in repeated cross-sectional surveys between 1985 and 2010. Passive surveillance of malaria cases was maintained until end 1999. Bed nets were distributed after the surveys 1993, 1999 and 2010.

Results: In 1985, overall parasite prevalence by microscopy was 70\% (90\% in children ages two to nine years). The prevalence decreased gradually by microscopy (38.9\% 1994, 26.7\% 1999) and msp2-PCR (58.7\% 1994, 44.8\% 1999), whereas real-time PCR prevalence remained higher throughout the 1990s (69.4\% 1994, 64.8\% 1999). In 2010, parasite prevalence was $17.8 \%$ by real-time PCR and $16.3 \%$ by msp2-PCR, and estimated to $4.8 \%$ by microscopy. Spleen rates in children ages two to nine years decreased earlier than parasite prevalence, from $>75$ to $42 \%$ in the 1980 s, to nil during the 1990s. The prevalence of severe and moderate anaemia decreased from 41.1 to $13.1 \%$. No deaths at the time of acute malaria were recorded when the research team lived in the village.
\end{abstract}

Conclusions: A marked decline in malaria transmission was observed over 25 years. The decrease was detected after the arrival of the research team and continued gradually both before and after distribution of bed nets. Spleen rates and microscopy identified early changes when transmission was still intense, whereas real-time PCR was a more sensitive metric when transmission was reduced. The study provides historical data on malaria within a closely monitored rural village and contributes to the understanding of changing epidemiology in sub-Saharan Africa.

Keywords: Malaria, Parasite prevalence, PCR, Microscopy, Spleen, Epidemiology, Transmission, Tanzania

\footnotetext{
* Correspondence: anna.farnert@ki.se

Deceased

'Infectious Diseases Unit, Department of Medicine Solna, Karolinska Institutet,

Stockholm, Sweden

Full list of author information is available at the end of the article
} 


\section{Background}

Malaria remains a major global health problem. The infection is spread in tropical and subtropical regions with the most intense transmission in sub-Saharan Africa. Several countries have reported reduced transmission over the last decade [1]. Understanding the temporal changes in malaria transmission, determining factors that have contributed to these changes, and identifying optimal monitoring tools are important in designing future control interventions [2].

The Nyamisati Malaria Research Project was set up in 1985 in a fishing village in Rufiji District, Tanzania. A medical team established a health clinic in this community, which previously lacked primary health care, and became interested in understanding the impact of malaria. A research project, in which all villagers were invited, aimed to describe the epidemiology and clinical aspects of malaria longitudinally. The first cross-sectional surveys in 1985-1988 revealed a holo-endemic setting with 90\% parasite prevalence and $>75 \%$ spleen rates in two to nine years old children [3]. Repeated cross-sectional surveys were performed and a passive surveillance system for malaria was maintained until year 2000 when the team moved. Studies conducted within the project have described various aspects of malaria, e.g., epidemiology (1985-1988) and clinical presentation [3-5]; impact of coinfections with measles, influenza and pertussis [6]; molecular epidemiology and dynamics of Plasmodium falciparum populations [7-10]; parasite genetics and evolution $[11,12]$; as well as immunology [13-16], and human immunogenetics $[17,18]$. Reports of decreasing transmission in other African settings [19-21] inspired the research team to return to the village in 2010 to perform a crosssectional survey to assess parasite prevalence.

This paper summarizes the epidemiology of malaria in Nyamisati village over 25 years. Long-term changes in $P$. falciparum prevalence were evaluated using microscopy and two PCR methods: species-specific real-time PCR and merozoite surface protein-2 (msp2) genotyping PCR. Moreover, spleen rates, haemoglobin levels and incidence of febrile malaria were assessed over time. The study shows a marked yet gradual decline in malaria transmission in Nyamisati, and demonstrates that different methods to monitor malaria endemicity are informative at different stages of changing transmission intensity.

\section{Methods}

\section{Study area and population}

Nyamisati village $\left(7^{\circ} 47^{\prime} \mathrm{S} ; 39^{\circ} 16^{\prime} \mathrm{E}\right)$ is situated in the Rufiji River Delta, the largest area of mangrove forest on the East African coast, in the Pwani region, Tanzania (Figure 1). The population is of Bantu origin and Muslim faith. Fishing and rice farming are the main occupations. Houses are mainly built in mud with roofs of grass and palm leaves. Malaria

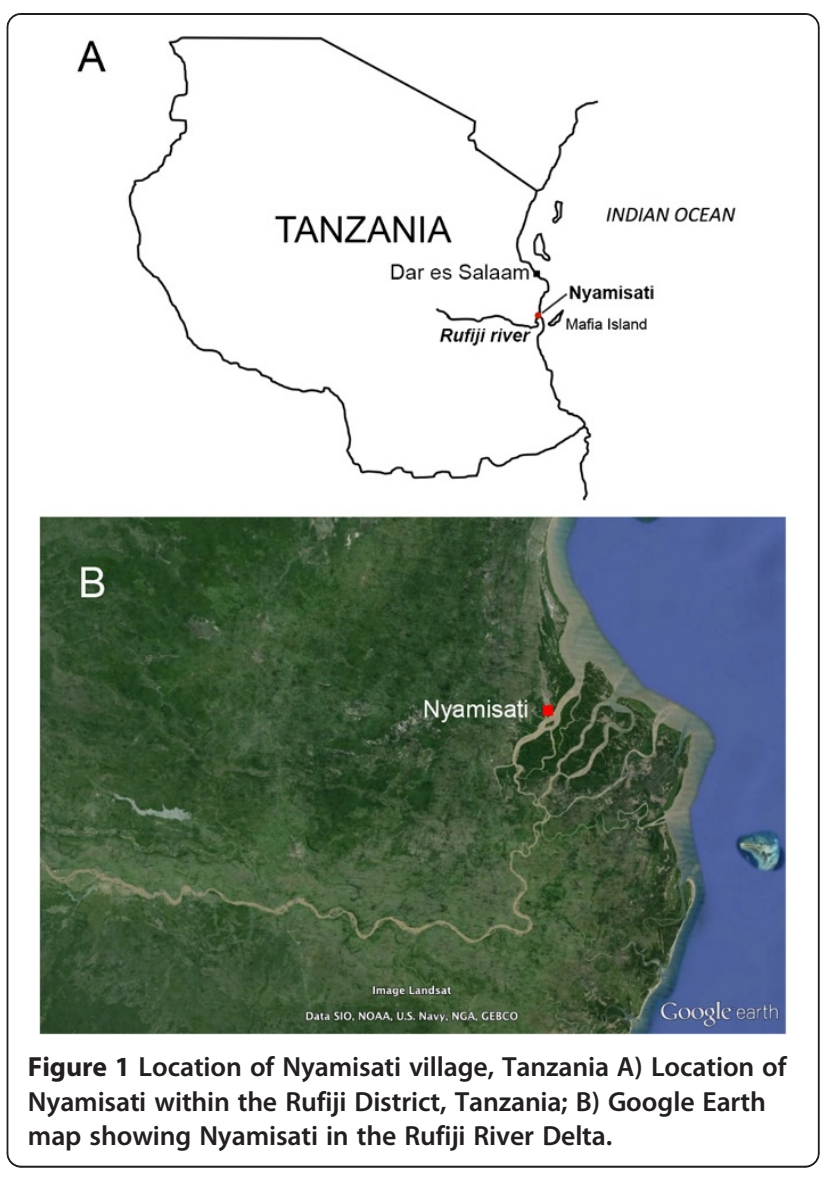

transmission is perennial with some seasonal fluctuations following the long and short rains, usually occurring in March-June and November-January, respectively (Additional file 1). HIV prevalence was $4.6 \%$ in adults in 1993. Sickle cell trait was detected by electrophoresis in $15 \%$ of villagers [3].

\section{Health care}

A team, including a physician, a nurse and two locally trained assistants lived in the village 1984-2000. A health clinic was continuously available for consultations free of charge. Except for a few traditional healers, there were no other health facilities in this or neighbouring villages at the time. The health care management was gradually handed over to a governmental rural medical aid (RMA). Emergencies and more complicated medical conditions were treated by the physician or referred to Mchukwi Hospital, $72 \mathrm{~km}$ away.

Malaria was diagnosed by microscopy and treated with chloroquine during the first years and later on with sulphadoxine-pyrimethamine (SP), alone or in combination with oral quinine. The different anti-malarial drugs administered at the health unit are summarized in Additional file 2. Artemether-lumefantrine (Coartem $\left.{ }^{\circ}\right)$ was, according to the RMA, available in the village from 2009. Drugs were administered free of charge through 
the health unit. There were no other drug outlets in the village before 2000 .

\section{Malaria control}

In 1985, 3\% of households owned a bed net. Between October 1993 and April 1994, 300 insecticide-treated bed nets (ITNs) were distributed to pregnant women and families with small children. The research team also distributed bed nets after the cross-sectional surveys in 1999 (900 ITNs not re-impregnated) and 2010 (1,000 long-lasting insecticide-treated nets (LLINs). The bed net coverage, assuming 1.8 individuals per net [22] was $4 \%$ in 1985 , $45 \%$ in $1993-1994$ and $100 \%$ after the survey in 1999. According to the villagers there was no other large-scale distribution of bed nets between 1999 and 2010. Indoor residual spraying and larval control were not used. Chemoprophylaxis with chloroquine and later intermittent preventive treatment (IPT) with SP were administered to pregnant women from a governmental maternal health unit. Except for a small study in 1986 [5], chemoprophylaxis and IPT were not administered to children.

\section{Malaria surveillance and cross-sectional surveys}

A passive surveillance system for malaria was maintained between 1986-1989 and 1993-1999. The villagers were instructed to come to the clinic in the event of fever. Individuals who presented with fever (axillary temperature $>37.5^{\circ} \mathrm{C}$ ), or history of fever ("hot body") within 24 hours, and had parasites detected by microscopy were administered anti-malarial treatment.

Cross-sectional surveys were performed in the population at the start of the long rainy season in 1986-1988, 1993-1999 and 2010. A subset of children aged ten to 19 years was sampled in January 2003 [11]. The crosssectional surveys consisted of blood sampling and a general health assessment, including an axillary temperature and auscultation of heart and lungs. A spleen examination was performed in children and enlarged spleens were graded according to Hackett's score [23]. Finger prick blood was collected for thick and thin films, Hemocue blood haemoglobin testing (Ängelholm, Sweden), and optional HIV testing. Venous blood was collected in $4 \mathrm{ml}$ EDTA tubes (for small children finger pricks in capillary tubes) and stored frozen as plasma and packed cells.

\section{Detection of malaria parasites by microscopy}

Thick and thin films were stained with Giemsa and analysed by conventional light microscopy. Parasite densities were estimated per microlitre $(\mu \mathrm{l})$ of blood and enumerated against the number of leukocytes assuming 8,000 leukocytes/ $\mu$ l. Blood films were considered negative if no parasites were detected in 100 microscopic fields of the thick smear.

\section{Detection of Plasmodium falciparum parasites by PCR}

Parasite prevalence was assessed by PCR at the crosssectional surveys 1994, 1995, 1999, and 2010 when most complete surveys across ages were performed. DNA was purified from frozen packed cells by Qiagen blood mini kit (Qiagen) (1994, 1995), phenol-chloroform extraction (1999), and using an automated Qiagen BioRobot ${ }^{\circ}$ M48 (2010). Real-time PCR for detection of Plasmodium species was performed by a multiplex probe-based method [24] using the ABI TaqMan 7500, with a cut-off of $40 \mathrm{cy-}$ cles to define positive samples. Genotyping of the $P$. falciparum msp 2 gene was performed by nested PCR using fluorescently labelled primers targeting the two allelic types of $m s p 2$ in separate nested reactions, fragment sizing by capillary electrophoresis in a DNA sequencer (Applied Biosystems), and data analysis by GeneMapper software [25]. Data on msp1 block 2 genotyping PCR from 2003 was available from a previous study [11]. The genotyping results are included here only with regard to detection of $P$. falciparum.

\section{Data analysis}

Data analysis was performed using GraphPad Prism 5.0 (GraphPad Software Inc, USA) and Stata 13.0 (StataCorp, USA). The data from 1985-1988 were retrieved from summarized data $[3,4]$. Parasite prevalence includes data from microscopy, $m s p 2-\mathrm{PCR}$ and real-time PCR. Missing prevalence data for $m s p 2-P C R$ in 1986 and 1993, as well as for microscopy in 2010 (due to staining artefacts after storage), were estimated using the prevalence estimation tool developed by Okell et al., validated for nested PCR data from all age cross-sectional surveys [26]. Agreement between methods was evaluated with the kappa statistic, using the Landis and Koch classification. Generalized estimating equation (GEE) regression models were used to account for statistical dependency of repeated observations when estimating the association between survey year and parasite prevalence, prevalence of sub-microscopic infections, and incidence of malaria. Complete population censuses were not performed; annual malaria incidence was therefore estimated among the individuals participating in the respective surveys. GEE logistic and negative binomial regression models were used to analyse binary (parasite prevalence), and count (malaria incidence) outcome variables. For each outcome, association with survey year was evaluated by univariate and multivariate models, adjusting for the effect of potential confounders and/or interactions (i.e., age, sex and fever and/or clinical episode of malaria at the time of survey. Anaemia was classified in four categories (non-anaemic, mild, moderate, and severe anaemia) based on haemoglobin levels according to WHO age and sex-specific criteria [27]. To evaluate changes in the overall prevalence of anaemia with time, a binary 
outcome defined as presence or absence of any level of anaemia was analysed using a univariate GEE logistic regression model. A univariate multinomial logistic regression model with robust clustering was used to evaluate changes in the prevalence of the four different levels of anaemia with time. Results from the multinomial logistic regression analysis are presented as predicted probabilities with $95 \% \mathrm{CI}$.

\section{Ethical considerations}

All data and blood samples were collected with informed consent from each participant and/or their guardians. The project was approved by the Nyamisati Village Board, the Ethical Review Board of the National Institute for Medical Research in Tanzania and the Central Ethical Review Board in Stockholm, Sweden.

\section{Results}

The population of Nyamisati increased from 451 individuals in 1985 to 1,553 individuals in 1999, as recorded by the research team. By 2012, 2,350 individuals lived in the village according to the National Bureau of Statistics in Tanzania [28]. The demographics of the individuals participating in the respective surveys are outlined in Table 1.

\section{Parasite prevalence by microscopy and PCR}

The overall parasite prevalence by microscopy was $68.7 \%$ (95\% CI $64.5-72.7 \%$ ) in $1986-1988,46.3 \%$ (95\% CI $42.0-50.5 \%$ ) in $1993,38.9 \%$ (95\% CI $35.5-42.4 \%)$ in 1994 , and $26.7 \%$ (95\% CI 23.8-29.7\%) in 1999 (Figure 2). Species typing by microscopy defined $\geq 95 \%$ of parasite positive samples as $P$. falciparum in all surveys. In 2010, the microscopy prevalence was estimated at $4.8 \%(95 \%$ credible interval of prediction 3.6-6.3\%) from the corresponding msp2-PCR data (according to [26]).

Both PCR methods consistently detected more P. falciparum-positive individuals than microscopy. The agreement between the real-time PCR and $m s p 2$ PCR positivity was $80 \%$ whereas it was $57.4 \%$ with microscopy (Additional file 3). Real-time PCR detected higher parasite prevalence than msp2-PCR in all years, although the difference was less pronounced in 2010 (Figures 2, 3 and 4). The msp2-PCR prevalence in 1986, as estimated from microscopy prevalence in all ages by the method of Okell et al. [26], was $83.7 \%$ (95\% credibility interval of prediction $79.8-87.0 \%$ ). The msp2-PCR prevalence decreased in parallel with microscopy during the 1990s (1994 58.7\%, 95\% CI 54.762.2\%; 1999 44.8\%, 95\% CI 41.5-48.2\%), whereas real-time PCR positivity remained at similar levels (1994 69.4\%, 95\% CI 65.5-73.1\%; 1999 64.8\%, 95\% CI 61.1-68.4\%). In 2010, real-time PCR prevalence was 17.8\% (95\% CI 15.1-20.7\%) and $m s p 2-$ PCR $16.3 \%$ (95\% CI 13.7-19.1\%) (Figure 2).

\section{Asymptomatic parasite prevalence}

The proportion of individuals who were febrile or had history of fever at the time of the cross-sectional survey varied over the years, being lowest in 1993 (1.1\%) and highest in 1999 (17.0\%) (Table 1). The asymptomatic parasite prevalence was lower than the overall prevalence in all surveys, with the largest difference among children aged 0-16 years in 1999 (Figure 4B). Among asymptomatic individuals, the odds of an infection being sub-microscopic increased significantly from 1994 to 1999 (GEE logistic regression age adjusted OR 1.73, 95\% CI $1.20-2.49 \%)$.

\section{Parasite prevalence by age}

In two to nine years old children, the age group conventionally used to estimate malaria endemicity [29], the parasite prevalence by microscopy was $90 \%$ in 1985 , $75.2 \%$ in $1993,60.6 \%$ in 1994 , and $49.0 \%$ in 1999 (Figure 3). The prevalence by real-time PCR in this age group was $78.2 \%$ in $1994,77.2 \%$ in 1999 and $12.8 \%$ in 2010 (Figure 3).

The peak parasite prevalence by microscopy gradually shifted to older children. In 1994-1999, the peak prevalence was seen in children aged five to eight years by

Table 1 Characteristics of the study population at the respective cross-sectional surveys

\begin{tabular}{|c|c|c|c|c|c|c|c|c|c|c|}
\hline & 1986 & 1993 & 1994 & 1995 & 1996 & 1997 & 1998 & 1999 & 2003 & 2010 \\
\hline Population $^{a}, n$ & 500 & 1,125 & 1,295 & 1,396 & 1,458 & 1,424 & 1,507 & 1,553 & $\mathrm{n} / \mathrm{a}$ & $\mathrm{n} / \mathrm{a}$ \\
\hline Cross-sectional survey, $\mathrm{n}$ & 470 & 555 & 792 & 712 & 326 & 337 & 509 & 889 & 105 & 808 \\
\hline Female, n (\%) & $239(51.1)$ & $300(54.1)$ & $448(56.6)$ & $403(56.6)$ & $205(62.9)$ & $187(55.5)$ & $276(54.2)$ & $476(53.5)$ & $42(40.0)$ & $407(50.4)$ \\
\hline Age, y median (range) & $15(0-90)$ & $15(0-83)$ & $14(0-84)$ & $14(0-84)$ & $31(1-86)$ & $22(1-82)$ & $24(2-78)$ & $17(1-84)$ & $13(10-19)$ & $15(1-82)$ \\
\hline Children $\leq 16$ y, n (\%) & $260(55.3)$ & $296(53.3)$ & $435(54.9)$ & $391(54.9)$ & $38(11.7)$ & $142(42.1)$ & $183(36.0)$ & $438(49.3)$ & $91(86.7)$ & $431(53.3)$ \\
\hline Children 2-9 y, n (\%) & $124(26.4)$ & $154(27.7)$ & $233(29.4)$ & $209(29.4)$ & $10(3.1)$ & $29(8.6)$ & $42(8.2)$ & $243(27.3)$ & 0 & $186(23.0)$ \\
\hline Fever at survey, n (\%) & nd & $6(1.1)$ & $78(9.8)$ & $89(12.5)$ & $28(8.6)$ & $5(1.4)$ & $25(4.9)$ & $152(17.1)$ & $1(1.0)$ & $23(2.9)$ \\
\hline $\begin{array}{l}\text { Fever at survey, } 2-9 y \\
\text { n (\% of all febrile) }\end{array}$ & nd & $3(50)$ & $60(77.9)$ & $58(65.2)$ & $7(25)$ & 0 & $2(8)$ & $95(62.5)$ & 0 & $9(39.1)$ \\
\hline
\end{tabular}

${ }^{\mathrm{a}}$ The population size is based on the individuals registered in the research database at the respective years. 


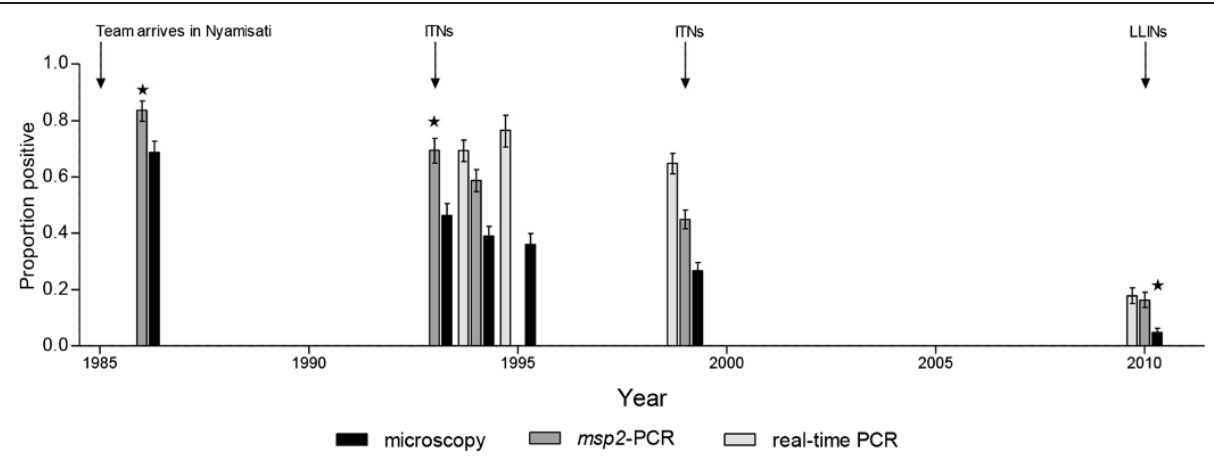

Figure 2 Parasite prevalence in Nyamisati 1985-2010 including all ages, by microscopy and two PCR methods (msp2 genotyping PCR and real-time species-specific PCR). The parasite prevalence by msp2-PCR for 1986 and 1993 as well as microscopy for 2010 were estimated from slide and msp2-PCR data, respectively, using the prevalence estimation tool developed by Okell et al. [26] (as indicated by *).

microscopy and nine to 12 years by PCR. In 2010, the peak PCR prevalence had shifted to the 13-16 years group (Figure 4). The proportion sub-microscopic infections (negative by microscopy and positive by real-time PCR) increased with age. For instance, in 1999, 9.0\% of asymptomatic individuals $>16$ years were parasite positive by microscopy and $51.3 \%$ by real-time PCR. The GEE logistic regression revealed that the odds of being parasite positive decreased with survey year in all age groups, with the most pronounced decrease in five to eight and nine to 12 years old children by real-time PCR (both OR 0.79, 95\% CI 0.76-0.83\%) (Table 2). In a subset of asymptomatic children in 2003, the parasite prevalence by microscopy was 3.1 and $13.6 \%$ in the ten to 12 and 13-16 years old children, respectively, and 46.9 and $46.6 \%$ by $m s p 1$-PCR [11].

\section{Spleen rates}

Enlarged spleens were recorded in $>75 \%$ of two to nine years old children in 1985, 62\% in 1986 and $42 \%$ in 1988 [3]. In 1993, spleen rate had decreased to $34.1 \%$ (95\% CI 26.8-42.2\%). During the following years, enlarged spleens were only sporadically recorded; and in 2010, $1.1 \%$ (95\% CI $0.1-3.8 \%$ ) of two to nine years old children had palpable spleens (Figure 3).

\section{Anaemia}

According to the GEE logistic regression analysis, the proportion of individuals with any level of anaemia decreased significantly over time, and compared to 1994 the odds of anaemia was lower in 1999 (OR 0.61, 95\% CI 0.47-0.79\%) and further reduced in 2010 (OR 0.32, 95\% CI 0.24$0.41 \%)$. Multinomial logistic regression analysis showed

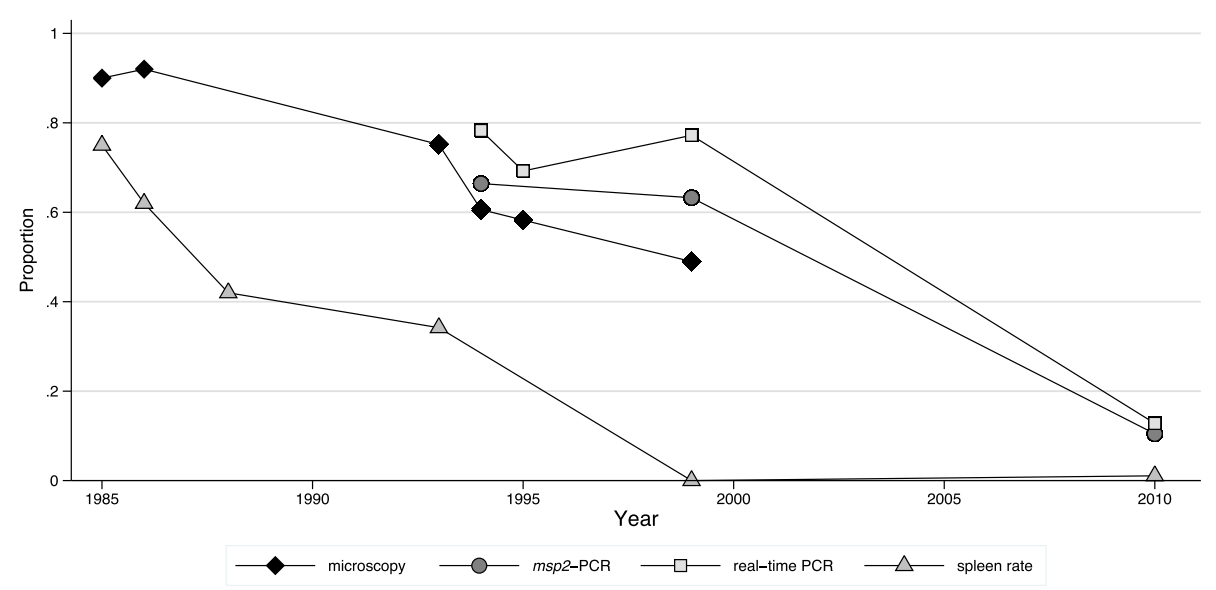

Figure 3 Parasite prevalence and spleen rates in children aged two to nine years in repeated cross-sectional surveys in 1985-2010. Parasite prevalence was assessed by microscopy, real-time PCR, and msp2-PCR. ITNs were distributed in October 1993-April 1994 ( $n=300)$ and in 1999 after the survey $(n=900)$; LLINs were distributed after the survey $2010(n=1,000)$ (as indicated by arrows). The data from 1986-1988 are available only as published data, spleen rates are available for individual years, whereas parasite prevalence by microscopy is only available as pooled data. 

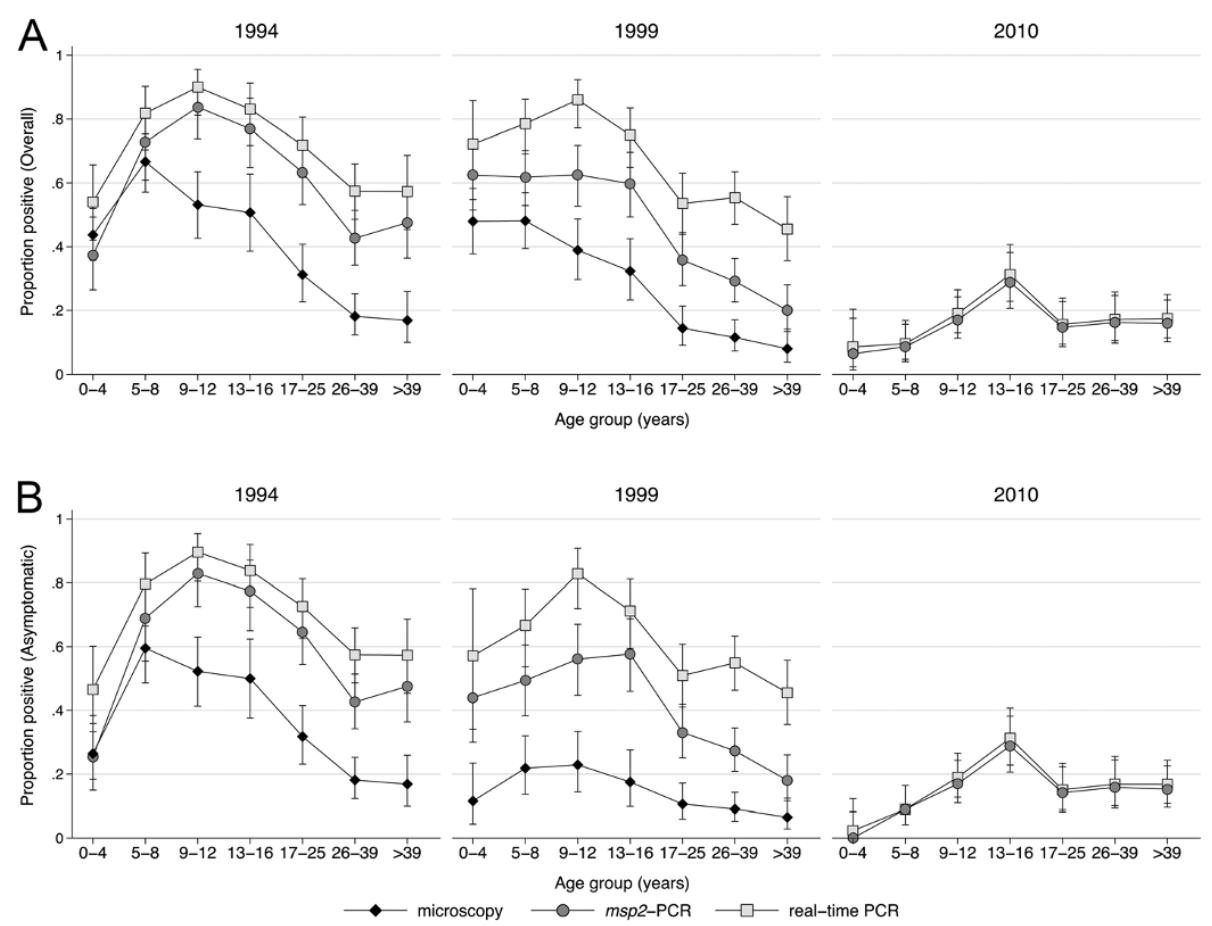

Figure 4 Age patterns of parasite prevalence by microscopy, real-time PCR, and msp2-PCR A) overall and B) in asymptomatic individuals in 1994, 1999 and 2010.

that the prevalence of severe and moderate anaemia decreased significantly from $41.4 \%$ (95\% CI 36.4-46.5\%) and $29.6 \%$ (95\% CI $24.9-34.3 \%$ ) in 1994 to $33.3 \%$ (95\% CI $30.2-$ $36.4 \%)$ and $24.8 \%$ (95\% CI 22.0-27.7\%) in 1999 and further to 27.6 (95\% CI $24.5-30.7 \%$ ) and $13.1 \%$ (95\% CI $10.7-$ $15.4 \%$ ) in 2010, respectively. Prevalence of mild anaemia remained stable throughout the period at $10.2 \%$ (95\% CI 7.1-13.3\%), 11.9\% (95\% CI 9.7-14.0\%) and 11.9\% (95\% CI 9.7-14.2\%) in 1994, 1999 and 2010, respectively (Figure 5).

\section{Malaria morbidity and mortality}

Malaria episodes were diagnosed year round with some seasonal fluctuations, largely following rainfalls (Figure 6A). Data on malaria incidence was available from 1986-88 and 1993-1999. Since a systematic demographic surveillance of the village was not performed, the exact size of the population is unknown. Therefore, estimates of malaria incidence were restricted to the individuals that participated in the survey and known to be in the village during the period of surveillance. Malaria incidence was highest among the youngest children and decreased with increasing age in all years of surveillance (Figure 6B, Additional file 4). The incidence of malaria was similar within the respective age groups in 1986-1988 and 1993-1995, with an increase in the late 1990s. In 1999, a large portion of episodes coincided with the cross-sectional survey (Figure 6A).
Parasite densities $>250,000 \mathrm{p} / \mu$ l were detected in $1.7 \%$ of 515 febrile malaria episodes $1986-88$ [3] and $0.6 \%$ of the 2,848 episodes in 1993-1999. Parasite densities in clinical malaria episodes varied between the years and increased from 1994 until 1998 (Figure 6C).

Severe anaemia was diagnosed in $1 \%$ of clinical malaria episodes in $1986-88$ and $0.4 \%$ in $1993-1999$. Seizures were reported in febrile children but there were no cases of unarousable coma. Other laboratory-based WHO criteria for severe malaria were not available. No deaths at the time of acute malaria were recorded when the research team lived in the village (1985-1991, 1993-2000).

\section{Discussion}

The Nyamisati Malaria Research Project provides longitudinal data on the epidemiology of malaria in a rural African village. A substantial, yet gradual, decline from holo- to hypo-endemicity was observed over 25 years, as reflected by changes in parasite prevalence from 90 to $<10 \%$ in two to nine years old children. Spleen rates decreased before changes in parasite prevalence were observed. Parasite prevalence by PCR, especially realtime PCR, remained high during the 1990s, and then revealed a substantial decrease by 2010 .

This study adds to a number of reports of changing malaria epidemiology, giving hope for elimination of malaria 
Table 2 Linear time-trend of odds of Plasmodium falciparum infection assessed by generalized estimating equation logistic regression models

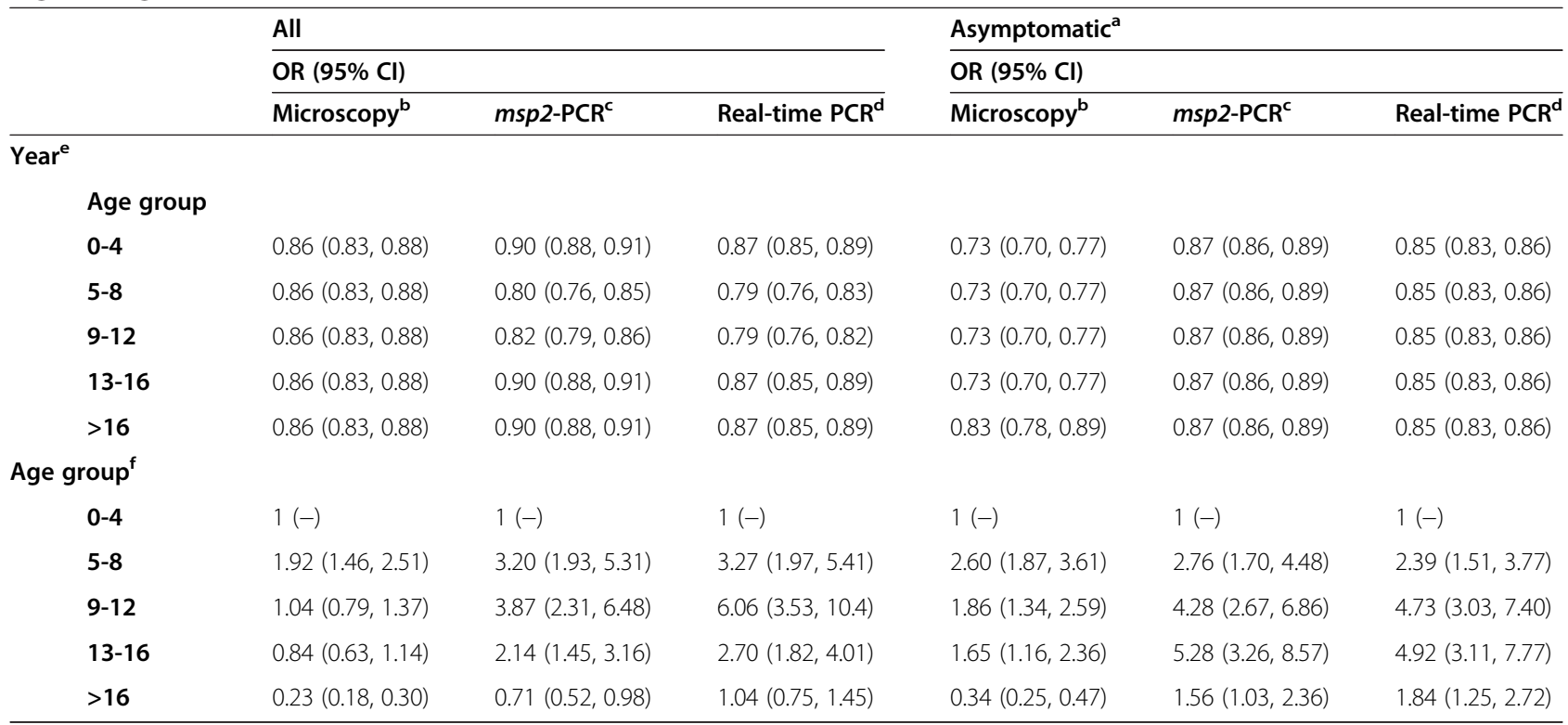

${ }^{a}$ Asymptomatic defined as absence of fever, history of fever or treatment at survey.

based on microscopy data from 1993-1999.

'Based on real-time PCR data from 1994-2010.

dBased on msp2-PCR data from 1994-2010.

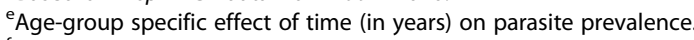

${ }^{f}$ Effect of age on parasite prevalence at baseline.

In the GEE logistic regression analysis year of survey was treated as a continuous variable and age group as a categorical variable in five categories (ages: 0-4, $5-8,9-12,13-16$, and $>16$ years). Due to significant interaction between year of survey and age group, effect of time on prevalence is presented as age group specific OR.

The odds of both overall and asymptomatic $P$. falciparum infection decreased with time and were most pronounced in asymptomatic individuals by microscopy. Abbreviations: $O R$ odds ratio, $\mathrm{Cl}$ confidence interval, $m s p 2$ merozoite surface protein 2 gene.

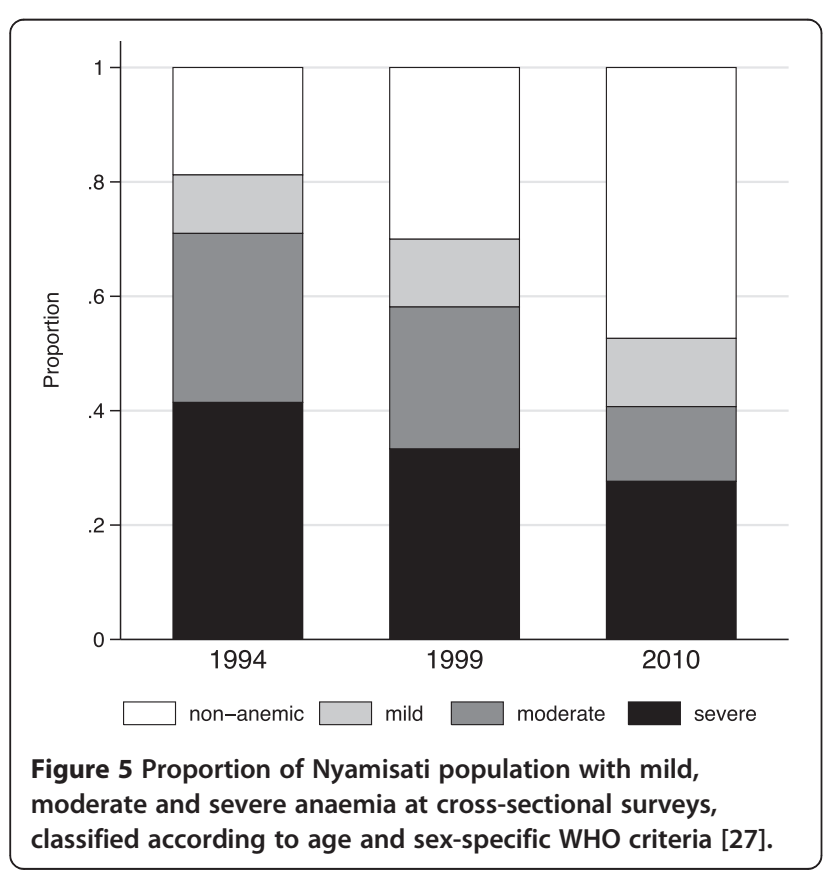

in some settings [1,19-21]. The long observation time contributes with historical data on malaria before the more recently introduced control interventions. Interestingly, very similar declines in malaria over the same time period have recently been reported from Muheza, Northern Tanzania [30] and Dielmo, Senegal [31].

The changes in transmission in Nyamisati were detected by different methods over time which demonstrates the advantage of using several metrics, i.e., spleen rates, microscopy and PCR in longitudinal surveillance. Molecular methods, especially PCR, have become increasingly used in epidemiological studies, and often reveal a large proportion of sub-microscopic infections [26]. The advantages and disadvantages of different methods to measure malaria transmission are well described in recent reviews (spleen rate not included) [32,33].

The first malariometric index to capture changes in the burden of malaria was spleen rate, which decreased down to nil while parasite prevalence by microscopy in the same two to nine age group remained above $75 \%$. Spleen rate (the proportion of children with enlarged spleens) was once the standard method to define malaria endemicity [29], but is no longer included in the 


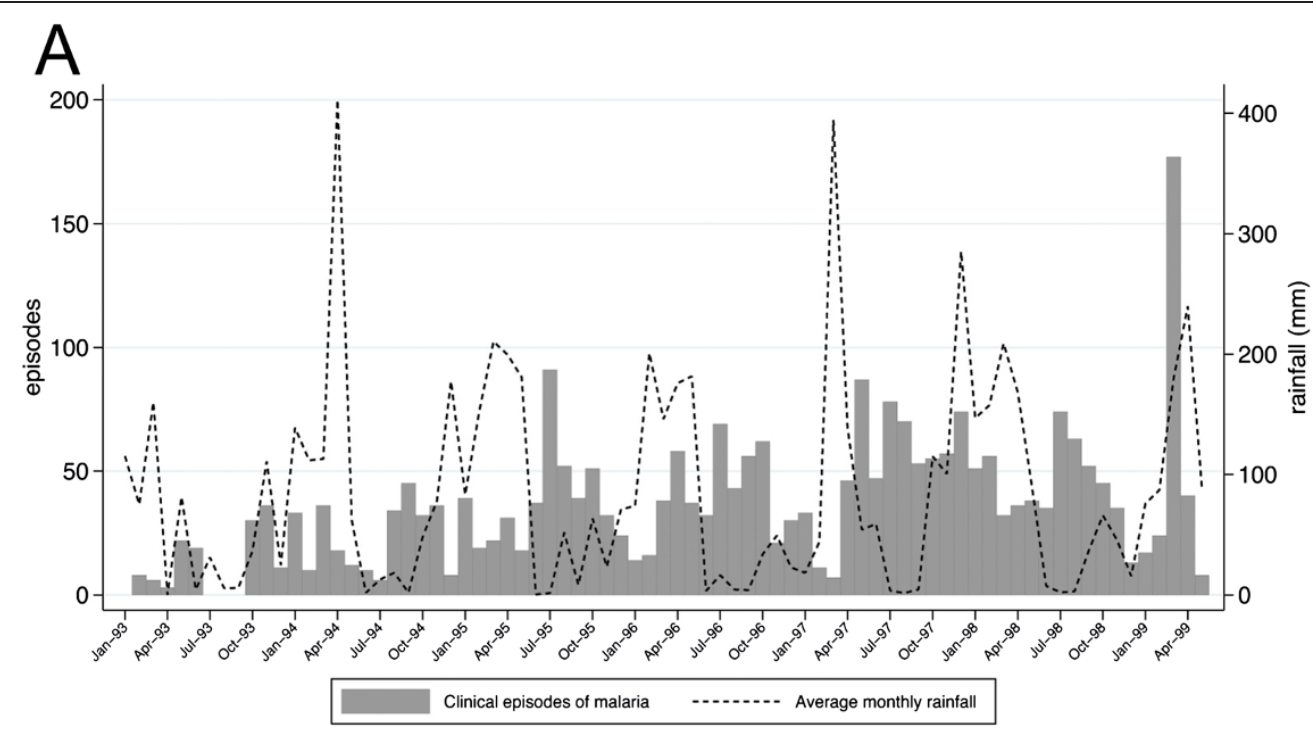

B

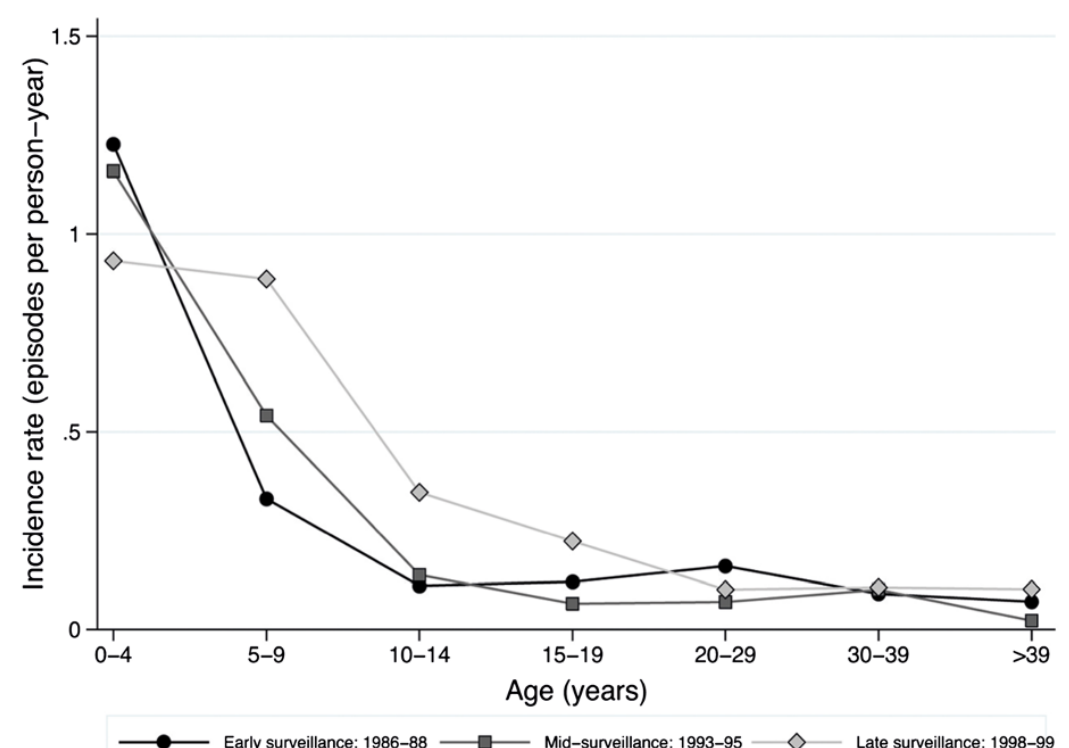

C

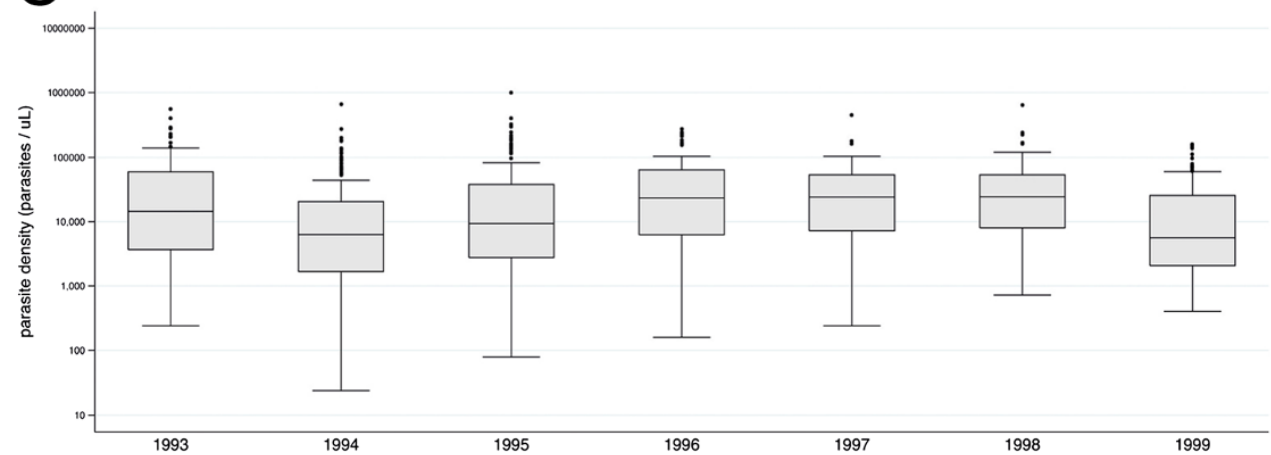

Figure 6 Clinical episodes of malaria. A) Number of clinical episodes of malaria diagnosed each month in 1993-1999 at the Nyamisati Health Clinic (bars) and rainfall patterns (solid line) averaged from data from the nearest meteorological stations of Utete, Kingupira, Kilwa Masoko, and Dar es Salaam International Airport, provided by the Tanzania Meteorological Agency; B) Incidence rate of clinical malaria by age over three observation periods 1986-1988, 1993-1995, and 1998-1999 (no data available 2010); C) Parasite densities in febrile malaria episodes over years. 
WHO guidelines for malaria surveillance, being replaced by assessments of parasite prevalence [34]. Although spleen size is unspecific for malaria on the individual level, spleen rates may still be useful as a screening tool on the population level. The data suggest that assessment of spleen size in combination with microscopy can detect early changes in areas of most intense transmission, e.g. after implementation of new control interventions. This is supported by similar decreases in spleen rates, down to nil, observed as transmission declined in the longitudinal studies in north-eastern Tanzania [30] and Senegal [31].

Microscopy showed a gradual decrease in parasite prevalence over time. Among asymptomatic individuals, microscopy detected an even more rapid decrease in parasite prevalence, indicating the value of defining the clinical status of individuals in cross-sectional epidemiological surveys.

The use of PCR was found most informative when transmission had decreased to a moderate or low level, as suggested in previous reviews [32,33]. PCR is well recognized to be a more sensitive method for parasite detection compared to microscopy, often revealing a large proportion of sub-microscopic infections in epidemiological studies [26]. This was also apparent here, especially as malaria declined in the 1990s. Furthermore, the choice of PCR method affects the interpretation of results on parasite prevalence. Including data from $m s p 2$ PCR allowed estimates of missing microscopy data according to the method by Okell et al., which is based on nested PCR data [26]. Real-time PCR had higher sensitivity than $m s p 2-\mathrm{PCR}$, likely due to differences in targeted genes (the multi-copy small subunit ribosomal RNA gene [35] versus the single copy $m s p 2$ gene [36]). Real-time PCR prevalence remained high during the 1990s and would not alone have revealed the changes in transmission detected by the other methods. The high number of sub-microscopic infections detected by realtime PCR likely contributed to ongoing transmission in this setting [37], demonstrating the importance of using sensitive molecular methods to monitor epidemiology at stages of decreasing transmission.

The age patterns of parasite prevalence changed over the study period. The age group with the highest prevalence gradually shifted to older children, suggesting reduced immunity as a result of lower exposure as previously described [38,39]. During the 1990s, increasing incidence of clinical malaria was observed which also might indicate a somewhat reduced immunity in the community as parasite prevalence declined during the 1990s. Moreover, a large number of symptomatic infections coincided with the cross-sectional survey in 1999, possibly reflecting changes to a more seasonal pattern of malaria transmission. The increasing incidence might, however, also reflect changes in drug susceptibility with increasing SP resistance. A similar trend was reported in Senegal before incidence eventually decreased in 2000s [31].

Cases of severe and fatal malaria were remarkably rare in the village. The continuous presence of a physician could have contributed to the paucity of severe malaria. However, malaria mortality was also low at the referral hospital in Mchukwi [40] and absence of malaria-specific mortality has been described in other settings of high transmission [31,41]. Changes in transmission might have altered incidence and severity of malaria in the village as a result of lower exposure to parasites. Malaria case data was unfortunately not available after 2000. Nonetheless, the low parasite prevalence in 2010 reflects a level of unstable transmission with potential increased risk of malaria and severe malaria in all age groups $[20,33]$.

The proportion of individuals with severe and moderate anaemia declined over time. Increasing haemoglobin levels were also reported in the Pwani region in the Tanzania Demographic and Health Survey in 2010 [42]. There was no iron or vitamin supplementation programmes in Nyamisati. The decrease in parasite burden likely contributed to these changes since anaemia is an important consequence of symptomatic and asymptomatic P. falciparum infections [43].

Declining transmission has been associated with introduction of one or several interventions, such as ITN/LLIN and artemisinin-based combination therapy (ACT) $[19,31]$. However, in some areas, such as in Uganda, malaria morbidity has increased despite scaling-up of control interventions [44]. The distribution of ITN in 19931994 and 1999 might partly explain the decreasing trends in parasite prevalence in Nyamisati, whereas ACT was not available until 2009 and access was low in the Rufiji district [45]. Nonetheless, decline in parasite burden as reflected by spleen rates and microscopy prevalence was observed already in the 1980s, i.e. before any of these control interventions were implemented. Also in other settings, such as The Gambia [21] and Kenya [20], declining transmission has been observed before the expansion of malaria control.

Interestingly, the declines in parasite prevalence over two decades in north-eastern Tanzania [30] and Senegal [31] are similar to the pattern observed in Nyamisati, despite differences regarding types and timing of interventions. In those areas, the Anopheles vector populations decreased markedly [46], along with changing vector species distribution [47] and entomological inoculation rates [31]. Entomological data are unfortunately not available in Nyamisati. In the Rufiji River Basin, the predominant species within the malariatransmitting Anopheles gambiae complex were Anopheles gambiae s.s, Anopheles arabiensis and Anopheles merus in 2003-2004 [48]. Changes in rainfall or temperature might have impacted on the vector [49], nonetheless no apparent changing trends in these observations were registered in 
nearby weather stations in coastal Tanzania during this 25-year period (Additional file 1).

The presence of a research team closely monitoring malaria with prompt and efficacious treatment is likely to have contributed to reducing parasite prevalence in Nyamisati and other closely monitored communities [30,31]. Already one year after the medical services were established in Nyamisati, mothers reported that their children's health status had improved [3]. Nonetheless, the parasite prevalence continued to decrease after the research team left the village in 2000, suggesting that other factors contributed to the declining transmission. Also at a nearby Rufiji Demographic Surveillance Site (DSS), parasite prevalence decreased from 23 to $14 \%$ by microscopy during 2001-2006 [50]. Although the lack of data in Nyamisati between 2000 and 2010 is a limitation of the study, it is also interesting that transmission declined despite the research team not being present. A factor that could have contributed to the decline is improved socio-economic status [51]. The village was initially very remote and isolated. Transportation improved over the years with tarmac roads and daily busses that began coming to Nyamisati in 2004, as it became the main port for ferries to Mafia Island. Nevertheless, housing conditions in the village remained similar during the study period.

The study is limited by data not being equally available for all variables at all time points. Cross-sectional surveys only provide a snapshot of the malaria transmission and incidence data available for only part of the study period. Nonetheless, the major strength of the study is that the continuous malaria surveillance and cross-surveys were performed at the same time of the year (at the beginning of the rainy season) by the same physician and research team (including microscopy as well as clinical and spleen assessments), using the same sample collection tubes and processing, thus providing a unique consistency and comparability over this extended period of time. Moreover, only the survey years with complete data and samples available throughout the different ages were included in the analyses. Another limitation might be that DNA extractions were performed by different methods for the different years, which potentially could affect the sensitivity of detection. However, the PCR analyses were recently performed in a highly standardized fashion by the same individual. Moreover, the samples collected 1994 and 2010 were extracted by similar commercial kits (manual and automated, respectively) and can thus be considered highly comparable.

\section{Conclusion}

The study demonstrates declining malaria transmission in this East African village over 25 years. Moreover it demonstrates the value of using different methods to monitor malaria transmission. Spleen rates and microscopy detect early changes when transmission is still intense. Conversely, PCR, especially real-time PCR, does not detect initial changes in transmission, but robustly and sensitively reveals a large proportion of sub-microscopic infections as transmission declines in a population.

The gradually declining transmission appears to have started already in the 1980s, with the arrival of the research team in 1985, and continued gradually after that. From the present data it is not possible to determine how much the interventions with ITNs contributed to the decline, partly because of the lack of data between 2000 and 2010. Further studies, using sero-epidemiological tools might contribute to the understanding of patterns and timing of transmission changes in periods when parasite prevalence data is missing. The historical data on malaria within this longitudinally, closely monitored rural village contributes to the understanding of changing epidemiology in sub-Saharan Africa over the last decades.

\section{Additional files}

\begin{abstract}
Additional file 1: Monthly rainfall (solid line) and temperature patterns, including high (dashed line) and low (dotted line) temperatures, in southeast coastal Tanzania from 1980 to 2010. Data from meteorological stations of Utete, Kingupira, Kilwa Masoko, and Dar es Salaam International Airport, kindly provided by Tanzania Meteorological Agency.

Additional file 2: Type of anti-malarial drugs administered through the health and research unit during the 1990s.

Additional file 3: Agreement between real-time PCR results with microscopy and msp2-PCR.

Additional file 4: Malaria incidence in Nyamisati 1993-1999 evaluated using univariate and multivariate generalized estimating equation negative binomial regression models.
\end{abstract}

\section{Abbreviations}

EDTA: Ethylenediamine tetra acetic acid; GEE: Generalized estimating equation; HIV: Human immunodeficiency virus; IPT: Intermittent preventive treatment; ITN: Insecticide-treated nets; LLIN: Long-lasting insecticide-treated nets; msp1: Merozoite surface protein 1 gene; msp2: Merozoite surface protein 2 gene; PCR: Polymerase chain reaction; RMA: Rural medical aid; SP: Sulphadoxine-pyrimethamine.

\section{Competing interests}

The authors declare that they have no competing interests.

\section{Authors' contributions}

$I R$ and $A F$ designed the study. IR, AB and ZP were involved in setting up the cohort. IR, SJ, MJ, and LM conducted the field study. MVH, GW, JS, and KT performed the molecular analyses. $\mathrm{VY}, \mathrm{UH}$ and MB performed the statistical analysis. AF and $\mathrm{VY}$ wrote the paper with critical revision from the co-authors. All authors read and approved the final manuscript.

\section{Acknowledgements}

We are most grateful to the villagers of Nyamisati for their continuous involvement and interest in the project. We wish to thank all members of the research team for their dedication; Professor Wen Kilama for his continued support; Kerstin Engström, Marianne Lebbad, Berit Aydin Schmidt, Ayan Ahmed and Pernilla Pettersson for technical assistance; Michael White for valuable comments on the manuscript, Hemocue Ängelholm for providing devices for haemoglobin testing. We wish also to thank Seif 
Ahmad Kuchengo and Albina Chuwa at the Bureau of Statistics of Tanzania and Janet Loning'O at Tanzania Meteorological Agency.

This work was supported by the Swedish International Development Agency, the Pentecostal Swedish Mission, Radiohjälpen, and the Marianne and Marcus Wallenberg Foundation (grant number MMW2010.0067)

\section{Author details}

${ }^{1}$ Infectious Diseases Unit, Department of Medicine Solna, Karolinska Institutet, Stockholm, Sweden. ${ }^{2}$ Nyamisati Malaria Research, Rufiji, Tanzania. ${ }^{3}$ Osaka Institute of Technology, Osaka, Japan. ${ }^{4}$ Unit of Biostatistics, Department of Environmental Medicine, Karolinska Institutet, Stockholm, Sweden.

${ }^{5}$ Department of Parasitology and Entomology, Muhimbili University College of Health Science, Dar es Salaam, Tanzania. ${ }^{6}$ Department of Pathology, Aga Khan University Hospital, Nairobi, Kenya. ${ }^{7}$ Department of Microbiology and Tumour Biology, Karolinska Institutet, Stockholm, Sweden.

Received: 30 August 2014 Accepted: 17 November 2014 Published: 26 November 2014

\section{References}

1. WHO: World Malaria Report 2013. Geneva: World Health Organization; http://www.who.int/malaria/publications/world_malaria_report_2013/en/ Accessed August 12014

2. malERA Consultative Group on Monitoring, Evaluation, and Surveillance: A research agenda for malaria eradication: monitoring, evaluation, and surveillance. PLoS Med 2011, 8:e1000400.

3. Rooth I: Malaria Morbidity and Control in a Tanzanian Village. In PhD thesis. Karolinska Institutet; 1992. ISBN 9162804960.

4. Rooth I, Björkman A: Fever episodes in a holoendemic malaria area of Tanzania: parasitological and clinical findings and diagnostic aspects related to malaria. Trans R Soc Trop Med Hyg 1992, 86:479-482.

5. Rooth I, Sinani HM, Björkman A: Proguanil daily or chloroproguanil twice weekly are efficacious against falciparum malaria in a holoendemic area of Tanzania. Am J Trop Med Hyg 1991, 94:45-49.

6. Rooth I, Björkman A: Suppression of Plasmodium falciparum infections during concomitant measles or influenza but not during pertussis. Am $J$ Trop Med Hyg 1992, 47:675-681.

7. Färnert A, Snounou G, Rooth I, Björkman A: Daily dynamics of Plasmodium falciparum subpopulations in asymptomatic children in a holoendemic area. Am J Trop Med Hyg 1997, 56:538-547.

8. Färnert A, Rooth I, Svensson $\AA$, Snounou G, Björkman A: Complexity of plasmodium falciparum infections is consistent over time and protects against clinical disease in Tanzanian children. J Infect Dis 1999, 179:989-995.

9. Bereczky S, Liljander A, Rooth I, Faraja L, Granath F, Montgomery SM, Färnert A: Multiclonal asymptomatic Plasmodium falciparum infections predict a reduced risk of malaria disease in a Tanzanian population. Microbes Infect 2007, 9:103-110

10. Färnert A, Lebbad M, Faraja L, Rooth I: Extensive dynamics of Plasmodium falciparum densities, stages and genotyping profiles. Malar J 2008, 7:241

11. Tanabe K, Sakihama N, Rooth I, Björkman A, Färnert A: High frequency of recombination-driven allelic diversity and temporal variation of Plasmodium falciparum msp1 in Tanzania. Am J Trop Med Hyg 2007, 76:1037-1045

12. Tanabe K, Arisue N, Palacpac NMQ, Yagi M, Tougan T, Honma H, Ferreira MU, Färnert A, Björkman A, Kaneko A, Nakamura M, Hirayama K, Mita T, Horii T: Geographic differentiation of polymorphism in the Plasmodium falciparum malaria vaccine candidate gene SERA5. Vaccine 2012, 30:1583-1593.

13. Rooth I, Perlmann H, Björkman A: Plasmodium falciparum reinfection in children from a holoendemic area in relation to seroreactivities against oligopeptides from different malaria antigens. Am J Trop Med Hyg 1991, 45:309-318.

14. Bereczky S, Montgomery SM, Troye-Blomberg M, Rooth I, Shaw MA, Färnert A Elevated anti-malarial lgE in asymptomatic individuals is associated with reduced risk for subsequent clinical malaria. Int J Parasitol 2004, 34:935-942.

15. Reddy SB, Anders RF, Beeson JG, Färnert A, Kironde F, Berenzon SK, Wahlgren M, Linse S, Persson KEM: High affinity antibodies to Plasmodium falciparum merozoite antigens are associated with protection from malaria. PLoS One 2012, 7:e32242.

16. Rono J, Osier F, Olsson D, Montgomery S, Mhoja L, Rooth I, Marsh K, Färnert A: Breadth of anti-merozoite antibody responses is associated with the genetic diversity of asymptomatic Plasmodium falciparum infections and protection against clinical malaria. Clin Infect Dis 2013, 57:1409-1416.

17. Carpenter D, Abushama H, Bereczky S, Farnert A, Rooth I, Troye-Blomberg M, Quinnell RJ, Shaw MA: Immunogenetic control of antibody responsiveness in a malaria endemic area. Hum Immunol 2007, 68:165-169.

18. Carpenter D, Rooth I, Färnert A, Abushama H, Quinnell RJ, Shaw MA Genetics of susceptibility to malaria related phenotypes. Infect Genet Evol 2009, 9:97-103.

19. Bhattarai A, Ali AS, Kachur SP, Mårtensson A, Abbas AK, Khatib R, Al-Mafazy AW, Ramsan M, Rotllant G, Gerstenmaier JF, Molteni F, Abdulla S, Montgomery SM, Kaneko A, Björkman A: Impact of artemisinin-based combination therapy and insecticide-treated nets on malaria burden in Zanzibar. PLoS Med 2007, 4:e309.

20. O'Meara WP, Bejon P, Mwangi TW, Okiro EA, Peshu N, Snow RW, Newton CR, Marsh K: Effect of a fall in malaria transmission on morbidity and mortality in Kilifi, Kenya. Lancet 2008, 372:1555-1562.

21. Ceesay SJ, Casals-Pascual C, Erskine J, Anya SE, Duah NO, Fulford AJ, Sesay SS, Abubakar I, Dunyo S, Sey O, Palmer A, Fofana M, Corrah T, Bojang KA Whittle HC, Greenwood BM, Conway DJ: Changes in malaria indices between 1999 and 2007 in The Gambia: a retrospective analysis. Lancet 2008, 372:1545-1554.

22. Kilian A, Boulay M, Koenker H, Lynch M: How many mosquito nets are needed to achieve universal coverage? Recommendations for the quantification and allocation of long-lasting insecticidal nets for mass campaigns. Malar J 2010, 9:330.

23. Hackett LW: Spleen measurement in malaria. J Nat Malar Soc 1944, 3:121-123.

24. Shokoples SE, Ndao M, Kowalewska-Grochowska K, Yanow SK: Multiplexed real-time PCR assay for discrimination of Plasmodium species with improved sensitivity for mixed infections. J Clin Microbio/ 2009, 47:975-980.

25. Liljander A, Wiklund L, Falk N, Kweku M, Mårtensson A, Felger I, Färnert A: Optimization and validation of multi-coloured capillary electrophoresis for genotyping of Plasmodium falciparum merozoite surface proteins (msp1 and 2). Malar J 2009, 8:78.

26. Okell LC, Bousema T, Griffin JT, Ouédraogo AL, Ghani AC, Drakeley CJ: Factors determining the occurrence of submicroscopic malaria infections and their relevance for control. Nat Commun 2012, 3:1237.

27. WHO: Haemoglobin Concentrations for the Diagnosis of Anaemia and Assessment of Severity, Vitamin and Mineral Nutrition Information System. Geneva: World Health Organization; 2011. http://www.who.int/vmnis/ indicators/haemoglobin.pdf Accessed April 18, 2014.

28. National Bureau of Statistics Tanzania: Village Statistics-Census. 2012. www.nbs.go.tz Accessed April 18, 2014.

29. WHO: Terminology of Malaria and of Malaria Eradication: Report of a Drafting Committee. Geneva: World Health Organization; 1963. www.nbs.go.tz Accessed April 202014

30. Ishengoma DS, Mmbando BP, Segeja MD, Alifrangis M, Lemnge MM, Bygbjerg IC: Declining burden of malaria over two decades in a rural community of Muheza district, north-eastern Tanzania. Malar J 2013, 12:338.

31. Trape JF, Tall A, Sokhna C, Ly AB, Diagne N, Ndiath O, Mazenot C, Richard V, Badiane A, Dieye-Ba F, Faye J, Ndiaye G, Diene Sarr F, Roucher C, Bouganal C, Bassène H, Touré-Baldé A, Roussilhon C, Perraut R, Spiegel A, Sarthou IL, da Silva LP, Mercereau-Puijalon O, Druilhe $P$, Rogier C: The rise and fall of malaria in a west African rural community, Dielmo, Senegal, from 1990 to 2012: a 22 year longitudinal study. Lancet Infect Dis 2014, 14:476-488.

32. Tusting LS, Bousema T, Smith DL, Drakeley C: Measuring changes in Plasmodium falciparum transmission: precision, accuracy and costs of metrics. Adv Parasitol 2014, 84:151-208.

33. Hay S, Smith DL, Snow RW: Measuring malaria endemicity from intense to interrupted transmission. Lancet Infect Dis 2008, 8:369-378.

34. WHO: Disease Surveillance for Malaria Control. Geneva: World Health Organization; 2012. http://apps.who.int/iris/bitstream/10665/44851/1/ 9789241503341 eng.pdf Accessed April 20, 2014

35. Langsley G, Hyde JE, Goman M, Scaife JG: Cloning and characterisation of the rRNA genes from the human malaria parasite Plasmodium falciparum. Nucleic Acids Res 1983, 11:8703-8717.

36. Smythe JA, Coppel RL, Day KP, Martin RK, Oduola AM, Kemp DJ, Anders RF: Structural diversity in the Plasmodium falciparum merozoite surface antigen 2. Proc Natl Acad Sci U S A 1991, 88:1751-1755.

37. Lindblade KA, Steinhardt L, Samuels A, Kachur SP, Slutsker L: The silent threat: asymptomatic parasitemia and malaria transmission. Expert Rev Anti Infect Ther 2013, 11:623-639. 
38. Satoguina J, Walther B, Drakeley C, Nwakanma D, Oriero EC, Correa S, Corran P, Conway DJ, Walther M: Comparison of surveillance methods applied to a situation of low malaria prevalence at rural sites in The Gambia and Guinea Bissau. Malar J 2009, 8:274.

39. Mmbando BP, Vestergaard LS, Kitua AY, Lemnge MM, Theander TG, Lusingu JPA: A progressive declining in the burden of malaria in north-eastern Tanzania. Malar J 2010, 9:216.

40. Rooth I: Annual Report. Dar es Salaam, Tanzania: Mchukwi Hospital; 1984.

41. Maitland K, Williams TN, Peto TE, Day KP, Clegg JB, Weatherall DJ, Bowden DK: Absence of malaria-specific mortality in children in an area of hyperendemic malaria. Trans R Soc Trop Med Hyg 1997, 91:562-566.

42. National Bureau of Statistics Tanzania (NBS) and ICF Macro: Tanzania Demographic and Health Survey 2010. Tanzania: NBS; 2011. http://www.nbs. go.tz/takwimu/references/2010TDHS.pdf Accessed April 202014.

43. Abdalla $\mathrm{S}$, Weatherall $\mathrm{DJ}$, Wickramasinghe $\mathrm{SN}$, Hughes $\mathrm{M}$ : The anaemia of $P$. falciparum malaria. Br J Haematol 1980, 46:171-183.

44. Jagannathan P, Muhindo MK, Kakuru A, Arinaitwe E, Greenhouse B, Tappero J, Rosenthal PJ, Kaharuza F, Kamya MR, Dorsey G: Increasing incidence of malaria in children despite insecticide-treated bed nets and prompt anti-malarial therapy in Tororo, Uganda. Malar J 2012, 11:435.

45. Khatib RA, Selemani M, Mrisho GA, Masanja IM, Amuri M, Njozi MH, Kajungu D, Kuepfer I, Abdulla SM, de Savigny D: Access to artemisinin-based anti-malarial treatment and its related factors in rural Tanzania. Malar J 2013, 12:155.

46. Meyrowitsch DW, Pedersen EM, Alifrangis M, Scheike TH, Malecela MN, Magesa SM, Derua YA, Rwegoshora RT, Michael E, Simonsen PE: Is the current decline in malaria burden in sub-Saharan Africa due to a decrease in vector population? Malar $J$ 2011, 10:188.

47. Derua YA, Alifrangis M, Hosea KM, Meyrowitsch DW, Magesa SM, Pedersen EM, Simonsen PE: Change in composition of the Anopheles gambiae complex and its possible implications for the transmission of malaria and lymphatic filariasis in north-eastern Tanzania. Malar J 2012, 11:188.

48. Kigadye ES, Nkwengulila G, Magesa SM, Abdulla S: Diversity, spatial and temporal abundance of Anopheles gambiae complex in the Rufiji River basin, south-eastern Tanzania. Tanzan J Health Res 2010, 12:68-72.

49. Hay SI, Omumbo JA, Craig MH, Snow RW: Earth observation, geographic information systems and Plasmodium falciparum malaria in sub-Saharan Africa. Adv Parasitol 2000, 47:173-215.

50. Khatib RA, Skarbinski J, Njau JD, Goodman CA, Elling BF, Kahigwa E, Roberts JM, MacArthur JR, Gutman JR, Kabanywanyi AM, Smith EE, Somi MF, Lyimo T, Mwita A, Genton B, Tanner M, Mills A, Mshinda H, Bloland PB, Abdulla SM, Kachur SP: Routine delivery of artemisinin-based combination treatment at fixed health facilities reduces malaria prevalence in Tanzania: an observational study. Malar J 2012, 11:140.

51. Tusting LS, Willey B, Lucas H, Thompson J, Kafy HT, Smith R, Lindsay SW: Socioeconomic development as an intervention against malaria: a systematic review and meta-analysis. Lancet 2013, 382:963-972.

doi:10.1186/1475-2875-13-459

Cite this article as: Färnert et al: Epidemiology of malaria in a village in the Rufiji River Delta, Tanzania: declining transmission over 25 years revealed by different parasitological metrics. Malaria Journal 2014 13:459.

\section{Submit your next manuscript to BioMed Central and take full advantage of:}

- Convenient online submission

- Thorough peer review

- No space constraints or color figure charges

- Immediate publication on acceptance

- Inclusion in PubMed, CAS, Scopus and Google Scholar

- Research which is freely available for redistribution

Submit your manuscript at www.biomedcentral.com/submit
Ciomed Central 